Twins conceived following ART were admitted to NICU 17\% less frequently than the naturally conceived population in unadjusted admission rates. Adjustments for gestational age exacerbated this difference.

\section{PF.20 INTRAUTERINE TRANSFUSION FOR RHESUS ISOIMMUNISATION IN SCOTLAND}

\section{doi:10.1136/archdischild-2013-303966.032}

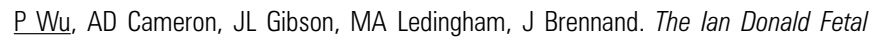
Medicine Unit, Southern General Hospital, Glasgow, UK

Scottish intrauterine transfusion (IUT) cases for severe Rhesus isoimmunisation over 2002-2011 were reviewed. One hundred and forty-one pregnancies underwent 437 IUTs (mean 3.09, range 1-8).

One hundred and thirty-one fetuses had middle cerebral artery Doppler peak systolic velocity values documented. All were $>1.5$ multiples of median prior to the first IUT, except for one that was inactive with a pericardial effusion. Twelve fetuses were hydropic. The haematocrit value prior to initial IUT was $20-29 \%$ in $38 \%$ of cases, whilst $27 \%$ had a haematocrit between $10-19 \%$. Initial IUT was most commonly performed between 29-32 weeks gestation (35\%) followed by 25-28 (26\%) and 21-24 (21\%) weeks gestation (range 17-25 weeks).

In the majority of cases, fetus was transfused via umbilical vein $(80 \%)$. Fourteen percent of transfusions were performed intrahepatically, 3\% intraperitoneally and 3\% were undocumented. Complications occurred in 58 (13\%) IUTs and include cord haematoma, difficult procedure, bradycardia or tachycardia necessitating unplanned delivery, and in utero death (5 fetuses). The procedurerelated loss rate was $1 \%$.

Birth outcomes were documented in 108 cases with a $94 \%$ live birth rate $(n=102)$. One patient underwent termination of pregnancy for trisomy 21. Short term postnatal outcomes were available for 86 neonates: 33 neonates required phototherapy only, with 31 cases requiring top-up transfusions. Nine neonates had an exchange transfusion and 9 had immunoglobulin or erythropoietin. Four neonates did not require any treatment. We conclude that our live birth and procedure-related loss rates are consistent with other published series. Parents need to be aware of potential postnatal therapies.

\section{PF.21 BRAIN ABNORMALITIES AND NEURODEVELOPMENTAL DELAY IN CONGENITAL HEART DISEASE: SYSTEMATIC REVIEW AND META-ANALYSIS}

doi:10.1136/archdischild-2013-303966.033

'A Khalil, ${ }^{2} \mathrm{~N}$ Suff, ${ }^{2} \mathrm{~A}$ Grande, ${ }^{1,3} \mathrm{~J}$ Carvalho, ${ }^{4} \mathrm{D}$ Cooper, ${ }^{1 B}$ Thilaganathan. ${ }^{1}$ St. George's University Hospital, London, UK; '2University College London, London, UK; ${ }^{3}$ Royal Brompton Hospital, London, UK; ${ }^{4} K i n g$ 's College London, London, UK

Objectives Studies have demonstrated an association between congenital heart disease (CHD) and neurodevelopmental delay, partly attributed to the risk of brain injury during cardiac surgery. However, neuroimaging studies have demonstrated a high incidence of preoperative brain abnormalities. The aim of this study was to perform a systematic review in order to quantify the non-surgical risk of brain abnormalities and neurodevelopmental delay in fetuses/newborns with CHD.

Methods MEDLINE, EMBASE and The Cochrane Library, without language restrictions were searched electronically, utilising combinations of the terms congenital heart, cardiac, neurologic, neurodevelopment, MRI, ultrasound, neuroimaging, autopsy, preoperative and outcome. Reference lists of relevant articles and reviews were hand searched for additional reports. Cohort and case-control studies were included. Case reports and editorials were excluded. Between-study heterogeneity was assessed using the $\mathrm{I}^{2}$ statistic.
Results The search yielded 9,129 citations. Full manuscripts were retrieved for 119, and 30 were included in the review and metaanalysis. 21 studies $(n=953)$ have reported brain abnormalities in fetuses, newborn or infants with CHD, either preoperatively or in those who did not undergo congenital cardiac surgery. The remaining 9 studies $(n=512)$ have reported preoperative data on neurodevelopmental assessment in newborn or infants with CHD. The prevalence of brain abnormalities was $36 \%$ (95\% CI, 26\%, 47\%; $\left.\mathrm{I}^{2}=90.5 \%\right)$ and of the neurodevelopmental delay $42 \%(95 \% \mathrm{CI}$, $34 \%, 51 \%$; $I^{2}=68.9 \%$ ), though with heterogeneity between studies. Conclusions In the absence of chromosomal or genetic abnormalities, fetuses with CHD are at increased risk of brain abnormalities and neurodevelopmental delay, which are independent of the surgical risk.

\section{PF.22 ESTABLISHING NORMOGRAMS FOR CERVICAL LENGTH IN PREGNANCY}

doi:10.1136/archdischild-2013-303966.034

A Khalid, JR Higgins, DJ McKenna. Cork University Maternity Hospital, Cork, Ireland

Objective To construct normograms for cervical length in lowrisk, singleton pregnancies from 14 weeks to 40 weeks gestation. Study design This was a prospective, cross-sectional study involving low-risk women attending Cork University Maternity Hospital conducted concurrently with a primary study to construct normograms for fetal biometry. Women were recruited in the first trimester and randomly allocated to a single scan between 14-40 weeks gestation. Scans were performed by a sole researcher. Gestation was calculated using the estimated due date assigned by dating scan. Recruits were Irish Caucasian women with a singleton pregnancy. Women with a previous history of preterm labour, mid-trimester pregnancy loss, cervical sutures, cone biopsy and large loop excision of transformation zone (LLETZ) were excluded. Measurements were obtained transvaginally using the Voluson E8 ultrasound by GE Healthcare.

Results Seven-hundred-and-ninety-three women were recruited into the primary study. Five-hundred-and-fifty-five women consented to cervical length measurements and were scanned as per protocol. Median maternal age was 32 (range 17-44). Median BMI was 24.7 (range 17.1-48.6). Nulliparous women constituted $46.5 \%$ (369/793) of recruits, $32.6 \%$ (261/793) were expecting their second child, $18.3 \%$ (145/793) were expecting their third or fourth child, while only $2.3 \%$ (18/793) were grand multiparous. Normograms for cervical length have been generated for this population. The following table demonstrates the calculated percentiles for each gestational week.

Conclusion We have constructed normograms for cervical length from 14 to 40 weeks gestation in the low-risk Irish Caucasian population.

\section{PF.23 MEDIUM TERM CHILDHOOD OUTCOME OF ISOLATED CONGENITAL TALIPES EQUINOVARUS DIAGNOSED ANTENATALLY}

doi:10.1136/archdischild-2013-303966.035

R Sharma, S Stone, A Alzeoubi, S Kumar. Queen Charlottes and Chelsea Hospitals, London, UK

Congenital talipes equinovarus (CTEV) is one of the most common developmental abnormalities affecting the lower limb. It may be associated with a variety of disorders and can be diagnosed antenatally using high-resolution ultrasound. Depending on severity, treatment usually involves passive manipulation of the affected joint or surgery. We investigated the medium-term childhood outcome of children diagnosed antenatally with isolated CTEV. Over a 